\title{
Day-Level Associations Between Substance Use and HIV Risk Behavior Among a Diverse Sample of Transgender Women
}

\author{
Brett M. Millar, ${ }^{1}$ Devin English, ${ }^{1}$ Raymond L. Moody, ${ }^{1,2}$ H. Jonathon Rendina, ${ }^{1-3, *}$ Demetria Cain, ${ }^{1,4}$
} Nadav Antebi-Gruszka, ${ }^{1,5}$ Joseph A. Carter, ${ }^{1,2}$ and Jeffrey T. Parsons ${ }^{1-3}$

\begin{abstract}
Purpose: Transgender women in the United States face elevated rates of HIV and of substance use. Studies measuring overall or aggregate levels of substance use have linked use to increased HIV transmission risk behavior (TRB). Although intensive longitudinal studies in other populations have found day-level links between substance use and TRB, no study has yet explored such links among transgender women. This study aimed to fill this gap in the literature.

Methods: Utilizing survey and 60-day timeline follow-back interview data from a sample of 214 transgender women in New York City, we tested whether day-level heavy drinking, marijuana use, and/or nonprescription stimulant use were associated with odds of engaging in any sex (vs. no sexual activity) or engaging in TRB (vs. sex without TRB), adjusting for overall levels of use.

Results: Multilevel models showed that each of the three substance types was associated with greater odds of engaging in sex on a given day-and more strongly so for heavy drinking among those with higher rates of heavy drinking, and for stimulant use among those with lower rates of stimulant use. Only marijuana use was associated with greater odds of TRB on a given day, but only among those with higher rates of use.

Conclusion: These findings substantiate day-level links between substance use and engaging in sexual activity among transgender women, and importantly, between marijuana use and greater likelihood of TRB on a day when sexual activity occurs. This highlights the importance of addressing substance use for sexual health among transgender women especially focusing on marijuana use.
\end{abstract}

Keywords: alcohol; drug use; harm reduction; intensive longitudinal data; prevention

\section{Introduction}

In efforts to better understand and address health inequities experienced by transgender women-defined as individuals assigned a male sex at birth who currently identify their gender as female-greater knowledge is needed regarding risk factors for adverse health outcomes. ${ }^{1}$ One prominent health inequity facing transgender women in the United States is elevated rates of HIV, with an estimated prevalence rate of $21.6 \%,{ }^{2,3}$ likely rooted in discrimination and oppression, and involving high rates of poverty, unemployment, and lack of social support. ${ }^{4-9}$ Accordingly, the need for a greater understanding of risk factors influencing HIV transmission risk behavior (TRB) among transgender women is paramount.

${ }^{1}$ Center for HIV/AIDS Educational Studies \& Training, Hunter College of the City University of New York (CUNY), New York, New York.

${ }^{2}$ Health Psychology and Clinical Science Doctoral Program, The Graduate Center of the City University of New York (CUNY), New York, New York.

${ }^{3}$ Department of Psychology, Hunter College of the City University of New York (CUNY), New York, New York.

${ }^{4}$ Community Health Sciences, University of Illinois-Chicago School of Public Health, Chicago, Illinois.

${ }^{5}$ Mental Health Counseling, Department of Psychology, City College of the City University of New York (CUNY), New York, New York.

Human Participant Protection: All study protocols were approved by the City University of New York (CUNY) Institutional Review Board. All participants provided their consent to take part in the study.

*Address correspondence to: H. Jonathon Rendina, MPH, PhD, Department of Psychology, Hunter College of the City University of New York, 695 Park Avenue, New York, NY 10065, E-mail: hrendina@hunter.cuny.edu

() Brett M. Millar et al. 2018; Published by Mary Ann Liebert, Inc. This Open Access article is distributed under the terms of the Creative Commons License (http://creativecommons.org/licenses/by/4.0), which permits unrestricted use, distribution, and reproduction in any medium, provided the original work is properly cited. 
Numerous cross-sectional studies have observed associations between overall or summed substance use indicators (e.g., averaged use over a given period, or any use vs. none) and rates of TRB among transgender women. ${ }^{10-16}$ This link between substance use and TRB is especially concerning given the findings of other studies showing that transgender women also have comparatively high rates of substance use. ${ }^{17-21}$

These cross-sectional findings resonate with extensive work that has focused on substance use as a risk factor in TRB among other populations such as heterosexual cisgender women and men ${ }^{22,23}$ and gay and bisexual men. ${ }^{24,25}$ However, research in these other populations has built upon these cross-sectional findings by studying associations between substance use on a given day and subsequent TRB events occurring on the same day. ${ }^{26-28}$ For example, Rendina et al. ${ }^{27} \mathrm{ob}-$ served day-level links between heavy drinking, marijuana, and club drug use and sexual behavior among gay and bisexual men, whereby use of each substance (especially club drugs) was associated with (1) increased odds of engaging in any sexual activity (though not necessarily TRB), and (2) increased odds of engagement in TRB (vs. sex that did not involve TRB) on that day. By including the individual's overall or aggregated level of use, analyses were able to show that the daylevel associations for club drug use and for marijuana were stronger among less frequent users compared with those who use more frequently. This kind of nuanced information highlights the value of intensive longitudinal analyses and is not possible in cross-sectional studies analyzing overall use. Unfortunately, day-level studies focusing on transgender women are scarce. To our knowledge, the only event-level study on substance use and TRB among transgender women is the study by Delgado et al. ${ }^{29}$ in Peru that combined gay and bisexual men with transgender women and only asked about alcohol use and the most recent sex event with up to three partners in the past 90 days.

Accordingly, we aimed to address this gap in the literature by examining whether substance use on a given day was associated with engagement in any sex and/or with engagement in sex involving TRB, among a diverse sample of transgender women in New York City. Specifically, we aimed to test day-level associations between heavy drinking (defined as having five or more drinks in the one sitting), marijuana, or nonprescription stimulant drugs on sexual engagement and sexual TRB. These variables are given in Table 1. Given the comparatively high rates of substance use
Table 1. Summary of Study Predictors and Outcomes

\begin{tabular}{lc}
\hline $\begin{array}{l}\text { Day-level predictor variables } \\
\text { Heavy drinking on a given day } \\
\text { Using marijuana on a given day }\end{array}$ & $\begin{array}{c}\text { Day-level outcomes } \\
\text { The odds of engaging } \\
\text { in any sex (vs. no sex) } \\
\text { Using stimulant drugs on a given day }\end{array}$ \\
$\begin{array}{c}\text { The odds of engaging in } \\
\text { sndividual-level predictor variables } \\
\text { Overall frequency of heavy drinking }\end{array}$ & $\begin{array}{c}\text { does not involve TRB) } \\
\text { Overall frequency of marijuana use } \\
\text { Overall frequency of stimulant drug use }\end{array}$ \\
\hline
\end{tabular}

TRB, transmission risk behavior.

generally observed among transgender women, we aimed to adjust for the individual's average level of use for each of the three substance types and to test whether the individual's average level of use moderated the day-level associations for each outcome. We hypothesized that, on days of substance use, the positive associations between use and both sexual engagement and sexual TRB would be even greater among transgender women with comparatively lower overall rates of use, and that the associations would still remain significant, although less strong, among transgender women with comparatively higher overall rates of use.

\section{Methods}

\section{Participants}

Participants were 214 transgender women from the New York City metropolitan area who completed baseline visits for a substance use and sexual risk behavioral intervention tailored to transgender women, Project T-Talk, between May 2014 and September 2016. For recruitment, we adapted a mix of active, passive, and online strategies that we have previously used to recruit gay and bisexual men. ${ }^{30-32}$ Active strategies included collaborating with community-based organizations, visiting venues, bars, and nightclubs, and building interpersonal connections and trust within local transgender communities through meetings and events. Additional passive recruitment efforts included distributing study recruitment materials to health clinics specializing in medical, mental health, and substance use treatment for transgender women. Online recruitment efforts consisted of emailing LISTSERVS, sending Project Newsletter emails to transgender women who had expressed interest in participating and advertising on social networking websites (e.g., Facebook, Craigslist). We also provided drop-in hours to maximize flexibility for participants to attend the initial baseline visit throughout the day and evenings. 
We screened a total of 487 transgender women, 382 (78\%) of whom met the criteria for study eligibility. These criteria included being 18 years old or older, being able to complete a survey in English, being a transgender woman (i.e., assigned a male sex at birth and identifying as female at the time of participation), providing contact information, living in New York City metropolitan area, and reporting at least one sexual act or one day of drug use in the past 60 days. We deemed participants ineligible if they were currently enrolled in a substance abuse treatment or an HIV risk or substance use intervention. Of the 219 transgender women who attended the baseline assessment, 214 completed all measures of interest and were included in the analytic sample for this study. The baseline assessment lasted between 70 and 150 min and consisted of a survey that assessed demographic characteristics and psychosocial factors (e.g., depressive symptoms), and a timeline follow-back (TLFB) interview (Sobell and Sobell, 1992) that assessed sexual behavior and substance use in the past 60 days (Irwin et al., 2006). Participants received $\$ 40$ for their participation in the baseline assessment. The Hunter College Institutional Review Board approved all study protocols.

\section{Measures}

Demographics. Participants self-reported their race and ethnicity, gender identity (i.e., whether participants consider themselves to be a transgender woman or a woman of transgender experience), relationship status (i.e., whether they are currently seeing someone they consider to be a main partner), sexual orientation identity (coded as heterosexual vs. lesbian, gay, bisexual, or queer; LGBQ), HIV status (with an HIV-negative status confirmed by testing or an HIV-positive status confirmed with documentation), annual income (in brackets of $\$ 10,000)$, level of education, current age, and the age when the participant first began living as a woman.

Behavioral variables. Participants completed a 60-day TLFB interview, ${ }^{33,34}$ indicating substance use and sex that occurred over the 60 days before the baseline assessment. For the present analyses, we focused on daily reports of heavy drinking (i.e., having five or more alcoholic drinks), use of marijuana, and use of nonprescription stimulants (i.e., cocaine/crack, ecstasy, methamphetamine), in line with the analytic plan of Rendina et al. ${ }^{27}$ For each day, we created three dichotomous indicators of whether the participant had engaged in heavy drinking, marijuana use, or nonprescription stimulants, and used these variables as daylevel (i.e., Level 1) indicators of substance use within our models. We also aggregated these three daily substance use indicators to the individual level to serve as global count variables indicating the number of days of use for each substance (i.e., Level 2).

For days on which a participant reported sex, we coded whether it was penetrative sex (i.e., involving vaginal or anal intercourse), whether condoms were used, whether it was with a casual partner or with a main partner who was of known discordant or unknown HIV status, and whether the event involved transactional sex (i.e., any exchange of sex for housing or money). We coded any act in which a participant had condomless penetrative sex with any casual partner and/or with a main partner of discordant or unknown HIV status as TRB. We did not collect information from all participants on whether they were using preexposure prophylaxis (PrEP) and also did not measure whether their partners were using PrEP, and were thus unable to include PrEP usage in our definition of TRB-however, PrEP use was very rare in the early stages of data collection and it is unlikely to have a substantial impact on results. To match the day-level substance use variables, we aggregated event-level sexual behavior data to the day level. Specifically, we created two variables to use as outcomes within our models: (1) a dichotomous indicator of whether any sexual activity occurred that day and (2) a trichotomous indicator of whether the participant engaged in no sex (coded as 0), sexual activity without TRB (e.g., mutual masturbation, oral sex, penetrative sex with a condom, condomless penetrative sex with a known seroconcordant partner; coded as 1), or sex involving TRB (coded as 2).

\section{Data analysis plan}

We began by examining descriptive statistics to characterize the demographic makeup of the sample. Next, we examined Spearman nonparametric correlations between the aggregated substance use count variables (i.e., days of heavy drinking, days of marijuana use, and days of nonprescription stimulant use) and the aggregate number of days having sex and sex involving TRB. We then ran a series of multilevel models, with day-level dichotomous indicators of heavy drinking, marijuana use, and nonprescription stimulant use at Level 1 and individual-level aggregated frequencies of use for each substance as a count variable at Level 2 . 
For the first outcome, whether or not participants engaged in any sexual activity on a given day, we used a binary logistic outcome (Model 1). For the second outcome, the trichotomous variable indicating whether the participant had no sex, had sex without TRB, or sex with TRB on a given day, we used a multinomial logistic model (Model 2). Although we used all data (both sex days and nonsex days) to calculate coefficients for Model 2, we focused our analysis on the portion of the model comparing sex days without TRB and TRB days. As such, we do not report comparisons with nonsex days in Model 2.

We ran both models with cross-level interactions between corresponding day-level and grand mean centered individual-level substance use (e.g., day-level heavy drinking by individual-level heavy drinking) to examine whether associations between day-level substance use and marginal probabilities for sexual engagement and TRB on a given day varied based on an individual's overall level of substance use. As such, these models included the following independent variables: day-level use of each of the three substance types (Level 1), aggregate individual-level use for each substance type (Level 2), and an interaction between corresponding substance categories on each level. We ran models with a random intercept, using an $\mathrm{AR}(1)$ covariance structure, and adjusted for HIV-positive status, relationship status, and day within the TLFB frame (i.e., 1 through 60 days). We also ran additional analyses adjusting for whether the sex event involved transactional sex to check if patterns remained consistent.

\section{Results}

Demographic characteristics are given in Table 2 . Approximately a third of the sample identified as Black/African American, 28\% identified as Latina, a quarter identified as white, $13 \%$ identified as multiracial, and the remaining five participants identified as Asian. A majority of participants had an HIV-negative status (65.4\%), had a yearly income below $\$ 20,000$ (80.4\%), and had an education level of some college or more $(57.9 \%)$. Approximately half of the participants reported being partnered (49.1\%) and LGBQ identified (52.8\%). Marijuana was the most frequently used substance with a mean use of 20.5 days and a median use of 7.0 days. Heavy drinking days $(M=5.4$, $S D=9.2$, median $=1)$ and nonprescription stimulant use days $(M=4.5, S D=10.1$, median $=0)$ were relatively less frequent during the 60-day period for most participants. The mean number of sex days was 12.6
Table 2. Demographic Characteristics and Prevalence of Outcomes Among Transgender Women in New York City $(N=214)$

\begin{tabular}{|c|c|c|}
\hline & $n$ & $\%$ \\
\hline \multicolumn{3}{|l|}{ Race/ethnicity } \\
\hline Black/African American & 69 & 32.2 \\
\hline Latina/Latinx & 60 & 28.0 \\
\hline White & 52 & 24.3 \\
\hline Multiracial & 28 & 13.1 \\
\hline Asian & 5 & 2.3 \\
\hline \multicolumn{3}{|l|}{ Gender identity } \\
\hline Transgender woman & 208 & 97.2 \\
\hline Other & 6 & 2.8 \\
\hline \multicolumn{3}{|l|}{ Relationship status } \\
\hline Single & 109 & 50.9 \\
\hline Partnered & 105 & 49.1 \\
\hline \multicolumn{3}{|l|}{ Sexual orientation } \\
\hline LGBQ & 113 & 52.8 \\
\hline Heterosexual/straight & 101 & 47.2 \\
\hline \multicolumn{3}{|l|}{ HIV status } \\
\hline Negative/unknown & 140 & 65.4 \\
\hline Positive & 74 & 34.6 \\
\hline \multicolumn{3}{|l|}{ Income } \\
\hline Below \$20K & 172 & 80.4 \\
\hline$\$ 20 \mathrm{~K}$ or more & 42 & 19.6 \\
\hline \multicolumn{3}{|l|}{ Education } \\
\hline High school or less & 90 & 42.1 \\
\hline Some college & 62 & 29.0 \\
\hline Bachelor degree & 49 & 22.9 \\
\hline \multirow[t]{2}{*}{ Graduate degree } & 13 & 6.1 \\
\hline & M & $S D$ \\
\hline Age (years; range 18-65) & 34.3 & 11.7 \\
\hline Age of first living as a woman $(n=208)$ & 22.4 & 10.0 \\
\hline Number of heavy drinking days (median $=1.0$ ) & 5.4 & 9.2 \\
\hline Number of marijuana use days (median $=7.0$ ) & 20.5 & 24.2 \\
\hline Number of stimulant use days (median $=0.0$ ) & 4.5 & 10.1 \\
\hline Number of days of any sexual activity (median $=8.0$ ) & 12.6 & 13.9 \\
\hline Number of sexual TRB days (median $=1.0$ ) & 4.3 & 10.1 \\
\hline
\end{tabular}

LGBQ, lesbian, gay, bisexual, queer.

$(S D=13.9)$ during the 60 -day period, with $85.5 \%$ of participants reporting at least two or more sex days. The mean number of TRB days was $4.3(S D=10.1)$, with $40.2 \%$ of participants reporting at least two or more TRB days.

\section{Aggregate-only analyses}

Table 3 provides bivariate correlations between aggregated sex days, TRB days, substance use days, and two covariates: HIV status and relationship status. We found that the number of sex days was positively correlated with total heavy drinking days, total stimulant use days, and being partnered-but not with marijuana use days. The number of TRB days was correlated only with total number of sex days. Aggregate levels of use among the three substances were positively correlated with each other such that, if a participant used one substance more frequently, they were more likely also to do 
Table 3. Bivariate Spearman Correlations Between Totals (of the Previous 60 Days) of Sex Days, Sexual TRB Days, Substance Use Days, and Covariates

\begin{tabular}{|c|c|c|c|c|c|c|c|}
\hline & 1 & 2 & 3 & 4 & 5 & 6 & 7 \\
\hline 1. No. of sex days & 1 & & & & & & \\
\hline 2. No. of sexual TRB days & $0.37^{* * *}$ & 1 & & & & & \\
\hline 3. No. of heavy drinking days & $0.28^{* * *}$ & 0.11 & 1 & & & & \\
\hline 4. No. of marijuana days & 0.10 & 0.02 & $0.16^{*}$ & 1 & & & \\
\hline 5. No. of stimulant drug days & $0.30 * * *$ & 0.08 & $0.31 * * *$ & $0.18^{* *}$ & 1 & & \\
\hline 6. HIV Status (Ref = HIV-negative) & 0.07 & 0.09 & -0.12 & $0.19^{* *}$ & $0.17^{*}$ & 1 & \\
\hline 7. Relationship status ( $R e f=$ Single) & $0.17^{*}$ & -0.12 & -0.06 & 0.04 & 0.07 & 0.07 & 1 \\
\hline
\end{tabular}

so for the other substances. Overall, marijuana use and stimulant use were more frequently common among HIV-positive participants. Relationship status was not associated with use of any of the three substances.

Simultaneous models of aggregate-level

and day-level substance use

Table 4 provides the two multilevel models predicting odds of sexual engagement versus no engagement (Model 1) and odds of sex with TRB versus sex without TRB (Model 2). In Model 1, day-level heavy drinking (adjusted odds ratio $[A O R]=2.30, p<0.001$ ), mari- juana use $(A O R=3.53, p<0.001)$, and stimulant use $(A O R=9.10, p<0.001)$ were all associated with significantly greater odds of sexual engagement (vs. no sex) on a given day. However, in Model 2, only day-level marijuana use $(A O R=2.69, p<0.001)$ was significantly associated with greater odds of engaging in TRB (vs. sex without TRB). Although day-level heavy drinking trended in the positive direction, suggesting greater odds of TRB, it did not reach statistical significance.

Regarding individual-level effects, aggregate number of stimulant use days $(A O R=1.05, p<0.01)$ was associated with greater odds of engaging in sex on a given day

Table 4. Multilevel Models Utilizing Day-Level and Individual-Level Substance Use to Predict Sexual Engagement and Sexual Transmission Risk Behavior

\begin{tabular}{|c|c|c|c|c|c|c|}
\hline & \multicolumn{3}{|c|}{ Model $1^{\mathrm{a}}$} & \multicolumn{3}{|c|}{ Model $2^{\mathbf{b}}$} \\
\hline & \multicolumn{3}{|c|}{ No Sex vs. Sex } & \multicolumn{3}{|c|}{ Non-TRB vs. TRB } \\
\hline & $b$ & AOR & $95 \% \mathrm{Cl}$ & $b$ & $A O R$ & $95 \% \mathrm{Cl}$ \\
\hline Intercept & -2.63 & $0.07^{* * *}$ & $0.05-0.11$ & -1.85 & $0.16^{* * *}$ & $0.07-0.34$ \\
\hline Day & -0.01 & $0.99^{* * *}$ & $0.99-1.00$ & 0.00 & 1.00 & $0.99-1.00$ \\
\hline HIV-status (Ref = HIV negative) & 0.17 & 1.18 & $0.71-1.97$ & 0.32 & 1.37 & $0.60-3.12$ \\
\hline In relationship (Ref = Single) & 0.68 & $1.97^{* *}$ & $1.23-3.16$ & -0.84 & $0.43^{*}$ & $0.20-0.93$ \\
\hline \multicolumn{7}{|l|}{ Level 1: day-level effects } \\
\hline Day-level heavy drinking & 0.83 & $2.30^{* * *}$ & $1.75-3.02$ & 0.32 & 1.38 & $0.84-2.27$ \\
\hline Day-level marijuana use & 1.26 & $3.53^{* * *}$ & $2.88-4.32$ & 0.99 & $2.69^{* * * *}$ & $1.64-4.41$ \\
\hline Day-level stimulant use & 2.21 & $9.10^{* * *}$ & $6.39-12.97$ & -0.19 & 0.83 & $0.46-1.51$ \\
\hline \multicolumn{7}{|l|}{ Level 2: individual-level effects } \\
\hline Frequency of heavy drinking & -0.02 & 0.98 & $0.95-1.01$ & 0.00 & 1.00 & $0.95-1.05$ \\
\hline Frequency of marijuana use & -0.01 & 0.99 & $0.97-1.00$ & -0.04 & $0.96^{*}$ & $0.93-0.99$ \\
\hline Frequency of stimulant use & 0.05 & $1.05^{* *}$ & $1.02-1.08$ & 0.04 & 1.04 & $0.99-1.08$ \\
\hline \multicolumn{7}{|l|}{ Day $\times$ individual effects } \\
\hline Day-level $\times$ frequency of heavy drinking & 0.07 & $1.07^{* * * *}$ & $1.05-1.10$ & -0.01 & 0.99 & $0.95-1.04$ \\
\hline Day-level $\times$ frequency of marijuana use & -0.01 & 0.99 & $0.98-1.00$ & 0.04 & $1.04^{*}$ & $1.00-1.08$ \\
\hline Day-level $\times$ frequency of stimulant use & -0.05 & $0.95^{* * *}$ & $0.93-0.97$ & -0.03 & 0.97 & $0.94-1.01$ \\
\hline
\end{tabular}

${ }^{*} p \leq 0.05$

${ }^{* *} p \leq 0.01$.

$* * * 0 \leq 0.001$.

aBinary logistic regression.

${ }^{\mathrm{b}}$ Multinomial logistic regression (only one of two comparisons are given). All models were adjusted for HIV status and relationship status and also day of data collection (i.e., day of timeline follow-back cycle).

AOR, adjusted odds ratio. 
(Model 1), indicating that those with more days of stimulant use in the 60-day period generally reported more days of engaging in sexual activity. In addition, aggregate marijuana use $(A O R=0.96, p<0.05)$ was associated with lower odds of engaging in TRB on a given day (Model 2). This indicates that those with more days of marijuana use in the 60-day period generally reported fewer events of TRB when sexual activity did occur.

We next examined the interaction effects between day-level substance use and individual-level substance use. In Model 1, we found that individual-level heavy drinking moderated the association between day-level heavy drinking and odds of engaging in sex $(A O R=1.07, p<0.001)$ - the positive association between day-level drinking and sexual engagement was higher among individuals who engaged in heavy drinking more often (Fig. 1). In the opposite direction, we found that individual-level stimulant use significantly moderated the association between day-level stimulant use and odds of engaging in sex $(A O R=0.95$, $p<0.001$ ), such that lower overall stimulant users had a greater increase in odds of sexual engagement on a day of use than those with more frequent use overall (Fig. 1).

In Model 2, we found that individual-level marijuana use moderated the positive association between day-level marijuana use and odds of engaging in TRB $(A O R=1.04, p<0.05)$. Although those with more frequent overall marijuana use tended to have lower odds of engaging in TRB on a sex day, the positive day-level association between marijuana use and their odds of TRB was stronger than for those with lower overall marijuana use (Fig. 2). Among those with lower overall marijuana use, the odds of engaging in TRB were not higher
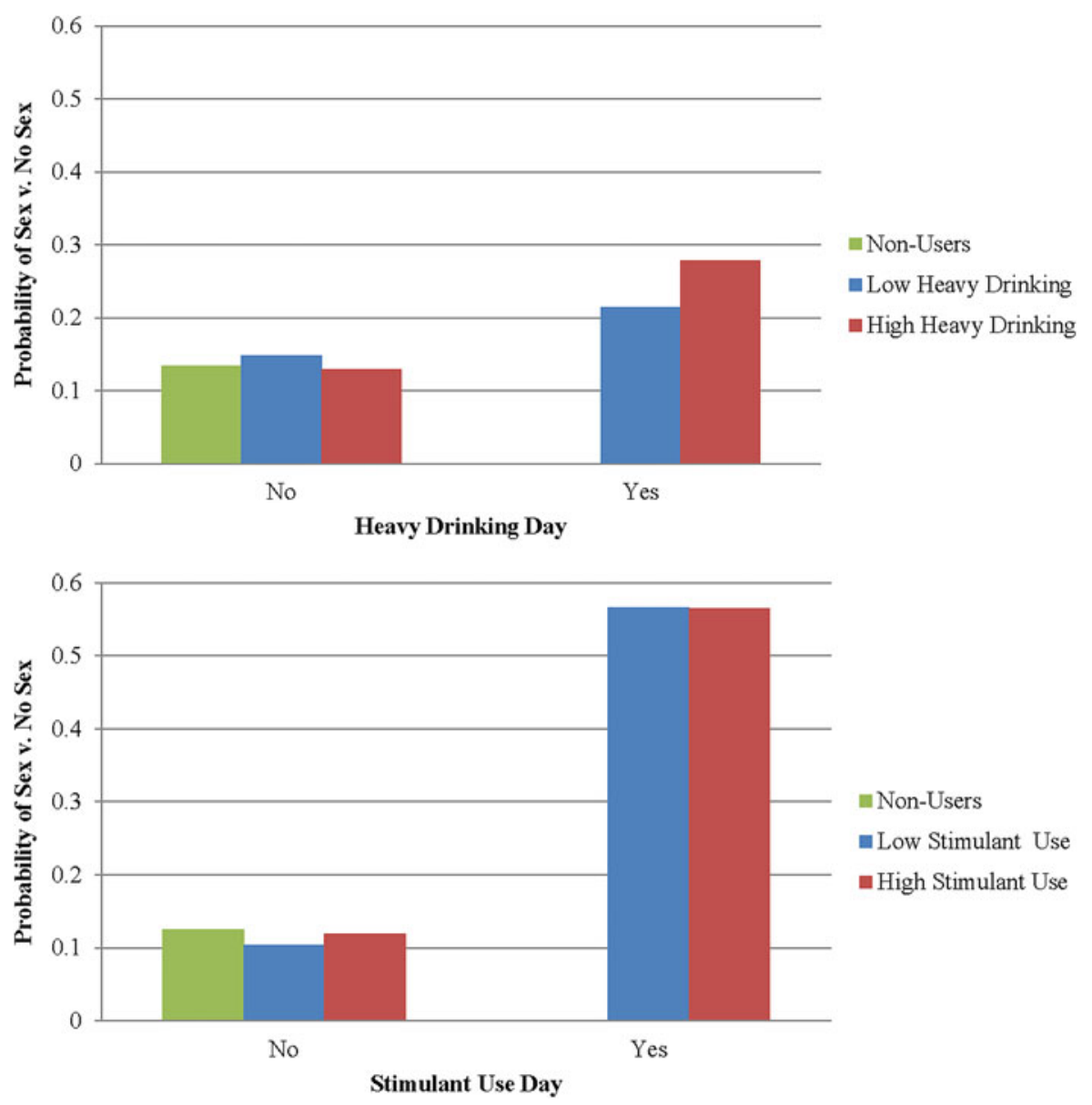

FIG. 1. Marginal probabilities of engaging in sexual activity based on individual and day-level use of substances. Note: Low substance use and high substance use are defined at the 25 th and 75 th percentiles, respectively. 


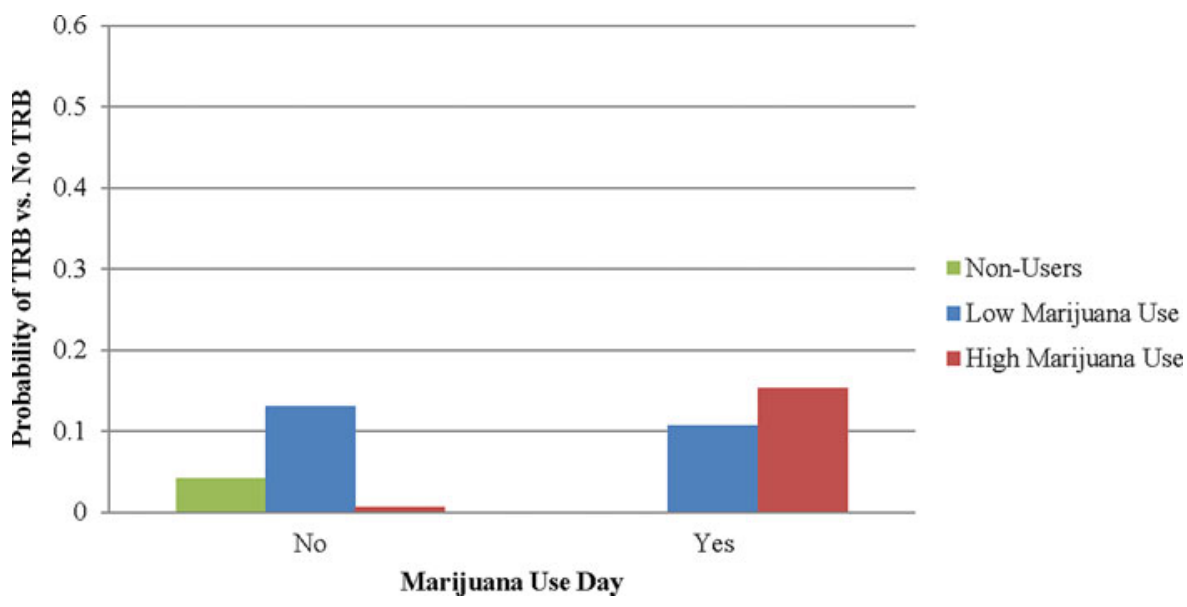

FIG. 2. Marginal probabilities of engaging in sexual TRB based on individual-level and day-level use of substances. Note: Low substance use and high substance use are defined at the 25th and 75th percentiles, respectively. TRB, transmission risk behavior.

on a day of marijuana use versus a day of no marijuana use. The patterns were not meaningfully altered in additional analyses when we adjusted for whether the event involved transactional sex.

\section{Discussion}

This study is, to our knowledge, the first to test for daylevel associations between substance use (here, heavy drinking, marijuana use, and/or nonprescription stimulant use) and sexual behavior focusing on transgender women. We found that, as hypothesized, use of any of these three substance types on a given day was associated with greater odds of engaging in sexual activity on that day (compared with not engaging in sexual activity) among a diverse sample of New York City-based transgender women. Indeed, heavy drinking was associated with more than twice the odds of sexual engagement on a given day, marijuana use was associated with $>3.5$ times the odds of sexual engagement, and stimulant use was associated with $>9$ times the odds of sexual engagement on that day. These findings that substance use and sexual activity often co-occur for transgender women are consistent with research on samples of cisgender women ${ }^{23}$ and gay and bisexual men, ${ }^{25,27}$ suggesting that substance use on a given day was a more informative predictor of sexual engagement compared with overall levels of use.

Our hypotheses regarding day-level associations between substance use and greater odds of sexual TRB, however, were only partially supported. Only mari- juana use on a given day was associated with greater odds of engaging in TRB compared with sex without TRB, at $>2.5$ times the odds. Although heavy drinking was in the hypothesized direction of increasing the odds of TRB, its local main effect did not reach statistical significance. The marijuana finding is consistent with evidence that marijuana use should be considered among the disinhibiting substances regarding sexual health, ${ }^{35}$ and suggests that interventions targeting the role of marijuana use on behaviors such as TRB among transgender women should be prioritized. However, the lack of significant findings for day-level heavy drinking and stimulant use predicting TRB in this study stands in contrast to previous studiesmostly examining overall frequencies of substance use-which have observed significant positive associations among transgender women ${ }^{10,15,16}$ and among other populations. ${ }^{26-28}$

Finally, we also tested whether these associations differed according to the individual's overall level of use. We found that the day-level association between heavy drinking and sexual engagement was stronger in those who engaged in heavy drinking more frequently, whereas the association between stimulant use and sexual engagement was stronger in those who engaged in less frequent use of stimulants overall. In terms of TRB, the day-level association between marijuana use and odds of TRB was stronger among those with greater overall marijuana use, counter to our hypothesis. These results suggest that the impact 
of marijuana on TRB on a given day is influenced by how frequently the substance is used by the individual in general.

This study begins to address a substantial gap in the literature on the influence of substance use on sexual behavior at the event-level among transgender women - and the findings have important implications in terms of HIV prevention efforts, particularly regarding marijuana use. Existing research on overall or aggregate substance use has found that transgender women who engage in more frequent use are at greater risk of engaging in TRB-suggesting that HIV prevention efforts should focus on transgender women who engage in more frequent substance use. ${ }^{1-15}$ Although our study cannot confirm the mechanism/s involved in the link between marijuana and TRB, our findings highlight the need to provide individuals frequently using marijuana with information and strategies to address links between their use and sexual risk behavior.

In terms of intervention implications, behavioral and motivational enhancement interventions have been shown to be effective at reducing substance use and sexual risk behavior among transgender women ${ }^{36}$ and gay and bisexual men. ${ }^{37,38}$ Some of these interventions incorporate a harm-reduction philosophy aimed at minimizing risk associated with substance use and sexual engagement. This study has implications for harm-reduction approaches to treatment for transgender women who may be resistant or ambivalent about totally eliminating their substance use. Among these, strategies may include reducing the quantity of use on a day of use, increasing self-efficacy for use of condoms or engaging in less risky sexual behavior, or increasing uptake of and adherence to medications (e.g., antiretroviral therapy; PrEP). The benefits of harm reduction on substance use and sexual health have been noted in other studies, such as a recent trial of a harm-reduction intervention that focused on gay and bisexual men that saw significant reductions in stimulant use, sexual partners, and receptive anal intercourse. ${ }^{39}$

The findings from this study should be considered in light of some limitations. First, the results of this study are based on a convenience sample who reported at least one recent sexual act or one recent drug use day, and thus these findings may not generalize to all transgender women. Second, this study utilized a TLFB interview to assess substance use and sexual behavior retrospectively. TLFB interviews are considered to be more accurate than other forms of retrospective recall but the risk of recall bias is still present. ${ }^{40}$ Although previous research has demonstrated relative consistency between retrospective and prospective assessments of substance use and sexual behavior, ${ }^{27} \mathrm{fu}-$ ture research should examine these associations using prospective assessment methods (e.g., ecological momentary assessment). Third, day-level drinking did involve consideration of quantity of use, but our measures of day-level marijuana use and stimulant drug use did not ask participants about the quantity of their use on a given day. Fourth, our analyses were not able to adjust for PrEP use by the participants' partners or for the detectability of either person's viral load in each sexual event. Furthermore, our definition of sexual TRB mainly pertains to risk for HIV transmission and did not address the possibilities for sexually transmitted infections occurring through other types of sexual activity (classified in this study as non-TRB sexual engagement). Future research may seek to explore the role of substance use on these other sexual health outcomes.

\section{Conclusions}

In sum, this study provides valuable, nuanced information about day-level links between substance use and sexual behavior among a sample of New York Citybased transgender women. In part, our findings highlight the need to distinguish between different types of substances when addressing substance use in relation to sexual risk behavior among transgender women, given the differing patterns observed. In addition, the tailoring of harm-reduction and contingencyplanning interventions according to the individual's overall level of use for each substance type is also recommended. Finally, it should be noted that a potential interpretation of our finding that both heavy drinking and stimulant use were positively associated with engaging in some sexual activity but not with engaging in TRB sex could be that, despite heavy drinking or stimulant use on a given day, many of the transgender women in our sample were able to navigate sexual activity with a partner without engaging in TRB. Further explorations of mechanisms and strategies used in these situations to prevent or avoid TRB may yield important insights for the sexual health of this population.

\section{Acknowledgments}

The authors acknowledge other members of the T-Talk Study Team (especially Ruben Jimenez, Tyrel Starks, 
Ana Ventuneac, Jonathan López Matos) and other former and current staff from the Center for HIV/AIDS Educational Studies and Training (Evie Arroyo, Julia Bassiri, Aaron Breslow, Alex Brousset, Ericka Florenciani, Miasha Forbes, Joshua Guthals, Ida Hammer, Chris Hietikko, Savannah Hornback, Doug Keeler, Kevin Jones, Tina Koo, Jonathan Lassiter, Jaisyn Melenciano, Will Mellman, Chloe Mirzayi, Chris Murphy, Vanessa Nasert, Carlos Ponton, Arjee Restar, Lena Saleh, Martez Smith, Laurie Spacek, Madison St. Claire, and Nala Toussaint) who were integrally involved in the development, implementation, and reporting of this study. The authors also thank the staff at CallenLorde Community Health Center (Asa Radix, Linda Li, Makada Bernard), and the LGBT Center in New York City (Carrie Davis and Cristina Herrera). Finally, special thanks to Drs. Richard Jenkins and Pamela Goodlow at NIDA.

T-Talk was funded by the National Institutes of Health [R01 DA 034661: H. Jonathon Rendina, Principal Investigator]. The content is solely the responsibility of the authors and does not necessarily represent the official views of the National Institutes of Health.

\section{Author Disclosure Statement}

No competing financial interests exist.

\section{References}

1. Wanta JW, Unger CA. Review of the transgender literature: where do we go from here?. Transgend Health. 2017;2:119-128.

2. Baral SD, Poteat T, Strömdahl S, et al. Worldwide burden of HIV in transgender women: a systematic review and meta-analysis. Lancet Infect Dis. 2013;13:214-222.

3. Centers for Disease Control and Prevention. HIV among transgender people. Available at www.cdc.gov/hiv/group/gender/transgender. Accessed 3 August, 2017.

4. Grant JM, Mottet LA, Tanis J, et al. Injustice at every turn: a report of the national transgender discrimination survey. Washington DC: The National Gay and Lesbian Task Force and the National Center for Transgender Equality. 2011. Available at www.thetaskforce.org/injustice-every-turn-reportnational-transgender-discrimination-survey/ Accessed November 1, 2018.

5. Nadal KL, Davidoff KC, Fujii-Doe W. Transgender women and the sex work industry: roots in systemic, institutional, and interpersonal discrimination. J Trauma Dissociation. 2014;15:169-183.

6. Poteat T, Wirtz AL, Radix A, et al. HIV risk and preventive interventions in transgender women sex workers. Lancet. 2015;385:274-286.

7. Sausa LA, Keatley J, Operario D. Perceived risks and benefits of sex work among transgender women of color in San Francisco. Arch Sex Behav. 2007;36:768-777.

8. Sevelius JM, Keatley J, Gutierrez-Mock L. HIV/AIDS programming in the United States: considerations affecting transgender women and girls. Women Health Issues. 2011;21:S278-S282.

9. UNAIDS. Global report: UNAIDS report on the global AIDS epidemic. 2012 Available at www.unaids.org/en/media/unaids/contentassets/documents/ epidemiology/2012/gr2012/20121120_UNAIDS_Global_Report_2012_with_ annexes_en.pdf. Accessed 30 June, 2017.

10. Brennan J, Kuhns LM, Johnson AK, et al. Syndemic theory and HIV-related risk among young transgender women: the role of multiple, co-occurring health problems and social marginalization. Am J Pub Health. 2012;102: $1751-1757$

11. Gilbert L, Raj A, Hien S, et al. Targeting the SAVA (substance abuse, violence, and AIDS) syndemic among women and girls: a global review of epidemiology and integrated interventions. J Acquir Immune Defic Syndr. 2015;69(Suppl 2):S118-S127.

12. Hotton AL, Garofalo R, Kuhns LM. Substance use as a mediator of the relationship between life stress and sexual risk among young transgender women. AIDS Educ Prev. 2013;25:62-71.

13. Neumann MS, Finlayson TJ, Pitts NL, Keatley J. Comprehensive HIV prevention for transgender persons. Am J Pub Health. 2017;107:207-212.

14. Operario D, Soma T, Underhill K. Sex work and HIV status among transgender women. J Acquir Immune Defic Syndr. 2008;48:97-103.

15. Parsons JT, Antebi-Gruszka N, Millar BM, et al. Syndemic conditions, HIV transmission risk behavior, and transactional sex among transgender women. AIDS Behav. 2018;22:2056-2067.

16. Reisner SL, Poteat T, Keatley J, et al. Global health burden and needs of transgender populations: a review. Lancet. 2016;388:412-436.

17. Benotsch EG, Zimmerman R, Cathers L, et al. Non-medical use of prescription drugs, polysubstance use, and mental health in transgender adults. Drug Alc Depend. 2013;132:391-394

18. Clements-Nolle K, Marx R, Guzman R, Katz M. HIV prevalence, risk behaviors, health care use, and mental health status of transgender persons: implications for public health intervention. Am J Pub Health. 2001;91: 915-921.

19. Nuttbrock L, Bockting W, Rosenblum A, et al. Gender abuse, depressive symptoms, and substance use among transgender women: a 3-year prospective study. Am J Pub Health. 2014;104:2199-2206.

20. Salazar LF, Crosby RA, Jones J, et al. Contextual, experiential, and behavioral risk factors associated with HIV status: a descriptive analysis of transgender women residing in Atlanta, Georgia. Int J STD AIDS. 2017;28: 1059-1066.

21. Santos GM, Rapues J, Wilson EC, et al. Alcohol and substance use among transgender women in San Francisco: prevalence and association with human immunodeficiency virus infection. Drug Alcohol Rev. 2014;33: 287-295.

22. Griffin JA, Umstattd MR, Usdan SL. Alcohol use and high-risk sexual behavior among collegiate women: a review of research on alcohol myopia theory. J Am College Health. 2011;58:523-532.

23. Wells BE, Kelly BC, Golub SA, et al. Patterns of alcohol consumption and sexual behavior among young adults in nightclubs. Am J Drug Alcohol Abuse. 2010;36:39-45

24. Bourne A, Reid D, Hickson F, et al. Illicit drug use in sexual settings ('chemsex') and HIV/STI transmission risk behaviour among gay men in South London: findings from a qualitative study. Sex Transm Infect. 2015; 91:564-568.

25. Parsons JT, Lelutiu-Weinberger C, Botsko M, Golub SA. Predictors of day-level sexual risk for young gay and bisexual men. AIDS Behav. 2013;17:1465-1477.

26. Feinstein BA, Newcomb ME. Event-level associations among drinking motives, alcohol consumption, and condomless anal sex in a sample of young men who have sex with men. AIDS Behav. 2017;21: 1904-1913.

27. Rendina HJ, Moody RL, Ventuneac $A$, et al. Aggregate and event-leve associations between substance use and sexual behavior among gay and bisexual men: comparing retrospective and prospective data. Drug Alcohol Depend. 2015;154:199-207.

28. Wray TB, Simons JS, Maisto SA. Effects of alcohol intoxication and autonomic arousal on delay discounting and risky sex in young adult heterosexual men. Addict Behav. 2015;42:9-13.

29. Delgado JR, Segura ER, Lake JE, et al. Event-level analysis of alcohol consumption and condom use in partnership contexts among men who have sex with men and transgender women in Lima, Peru. Drug Alc Depend. 2017;170:17-24.

30. Parsons JT, Vial AC, Starks TJ, Golub SA. Recruiting drug using men who have sex with men in behavioral intervention trials: comparison of internet and field-based strategies. AIDS Behav. 2013; 17:688-699.

31. Vial AC, Starks TJ, Parsons JT. Finding and recruiting the highest risk HIV-negative men who have sex with men. AIDS Educ Prevent. 2014;26: 56-67. 
32. Vial AC, Starks TJ, Parsons JT. Relative efficiency of field and online strategies in the recruitment of HIV-positive men who have sex with men. AIDS Educ Prevent. 2015;27:103-111.

33. Irwin TW, Morgenstern J, Parsons JT, et al. Alcohol and sexual HIV risk behavior among problem drinking men who have sex with men: an event level analysis of timeline followback data. AIDS Behav. 2006;10:299-307.

34. Sobell LC, Sobell MB. Timeline follow-back. In: Litten RZ, Allen JP (Eds). Measuring Alcohol Consumption. Totowa, NJ: Humana Press, 1992, pp. 41-72.

35. Simons JS, Maisto SA, Wray TB. Sexual risk taking among young adult dual alcohol and marijuana users. Addict Behav. 2010;35:533-536.

36. Glynn TR, van den Berg JJ. A systematic review of interventions to reduce problematic substance use among transgender individuals: a call to action. Transgend Health. 2017;2:45-59.

37. Parsons JT, John SA, Millar BM, Starks TJ. Testing the efficacy of combined motivation interviewing and cognitive behavioral skills training to reduce methamphetamine use and improve HIV medication adherence among HIV-positive gay and bisexual men. AIDS Behav. 2018;22:2674-2686.

38. Parsons JT, Lelutiu-Weinberger $C$, Botsko $M$, Golub SA. A randomized controlled trial utilizing motivational interviewing to reduce HIV risk and drug use in young gay and bisexual men. J Consult Clin Psychol. 2014;82:9-18.

39. Carrico AW, Flentje A, Gruber VA, et al. Community-based harm reduction substance abuse treatment with methamphetamine-using men who have sex with men. J Urb Health. 2014;91:555-567.
40. Griffith SD, Shiffman S, Heitjan DF. A method comparison study of timeline followback and ecological momentary assessment of daily cigarette consumption. Nicotine Tob Res. 2009;11:1368-1373.

Cite this article as: Millar BM, English D, Moody RL, Rendina HJ, Cain D, Antebi-Gruszka N, Carter JA, Parsons JT (2018), Day-level associations between substance use and HIV risk behavior among a diverse sample of transgender women, Transgender Health 3:1, 210-219, DOI: $10.1089 /$ trgh.2018.0032

\section{Abbreviations Used}

$\mathrm{AOR}=$ adjusted odds ratio

$\mathrm{LGBQ}=$ lesbian, gay, bisexual, or queer

$\operatorname{PrEP}=$ preexposure prophylaxis

TLFB $=$ timeline follow-back

$\mathrm{TRB}=$ transmission risk behavior

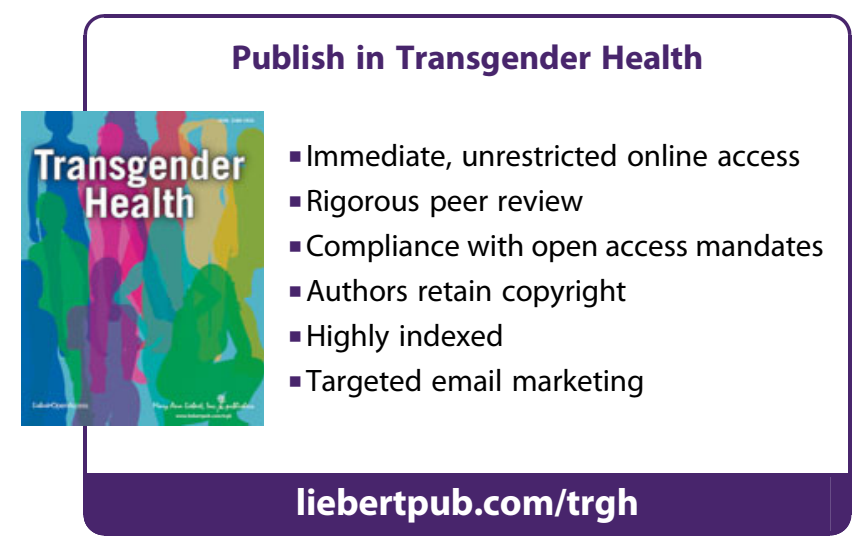

